\title{
Induction treatment in patients with stage III non-small cell lung cancer
}

\author{
Ramón Palmero $^{1,2 \#}$, Noelia Vilariño ${ }^{1,2 \#}$, Arturo Navarro-Martín ${ }^{3}$, Ernest Nadal ${ }^{1,2}$ \\ ${ }^{1}$ Department of Medical Oncology, Catalan Institute of Oncology, Barcelona, Spain; ${ }^{2}$ Clinical Research in Solid Tumors (CReST) Group, Oncobell \\ Program, Bellvitge Biomedical Research Institute (IDIBELL), Barcelona, Spain; ${ }^{3}$ Department of Radiation Oncology, Catalan Institute of Oncology, \\ Barcelona, Spain \\ Contributions: (I) Conception and design: All authors; (II) Administrative support: None; (III) Provision of study materials or patients: None; (IV) \\ Collection and assembly of data: All authors; (V) Data analysis and interpretation: All authors; (VI) Manuscript writing: All authors; (VII) Final \\ approval of manuscript: All authors. \\ \#These authors contributed equally to this work. \\ Correspondence to: Ernest Nadal. Department of Medical Oncology, Catalan Institute of Oncology, L'Hospitalet, Barcelona, Spain. \\ Email: esnadal@iconcologia.net.
}

\begin{abstract}
Stage III non-small cell lung cancer (NSCLC) comprises a highly heterogeneous group of patients defined according to the extent and localization of disease. Patients with discrete N2 involvement identified preoperatively with resectable disease are candidates for multimodal therapy either with definitive chemoradiation therapy, induction chemotherapy, or chemoradiotherapy (CTRT) followed by surgery. Neoadjuvant chemotherapy has yielded comparable survival benefit to adjuvant chemotherapy in patients with stage II-III disease and may allow for downstaging the tumor or the lymph nodes, an earlier delivery of systemic treatment, and better compliance to systemic therapy. The use of immune checkpoint inhibitors (ICIs) as induction therapy shows encouraging activity and a favorable safety profile in patients with resectable early stage or locally advanced NSCLC. An unprecedented rate of pathological response and downstaging has been reported in single-arm clinical trials, especially when immunotherapy is combined with neoadjuvant chemotherapy. Ongoing randomized phase II/III clinical trials assessing the efficacy and safety of induction with immunotherapy plus chemotherapy have the potential to establish this therapeutic approach as a novel standard of care. These trials aim to validate pathological response as a surrogate marker of survival benefit and to demonstrate that this therapeutic strategy can improve the cure rate in patients with stage II-III NSCLC.
\end{abstract}

Keywords: Locally advanced non-small cell lung cancer (locally advanced NSCLC); neoadjuvant treatment; induction therapy; resectable disease

Submitted Mar 21, 2020. Accepted for publication Jun 09, 2020.

doi: $10.21037 /$ tlcr-20-420

View this article at: http://dx.doi.org/10.21037/tlcr-20-420

\section{Introduction}

Locally advanced non-small cell lung cancer (NSCLC) is defined as a lung tumor that has spread to nearby tissue or lymph nodes (National Cancer Institute definition). According to the $8^{\text {th }}$ edition of the TNM lung cancer staging manual, locally advanced NSCLC comprises a wide range of clinical presentations including primary tumor extension into extrapulmonary structures (T3 or T4) or mediastinal lymph node involvement (N2 or N3), without presence of distant metastases (M0). Patients with extensive mediastinal N2 infiltration are considered unresectable and are candidates for non-surgical multimodality strategies. The standard of care for fit patients is concomitant 


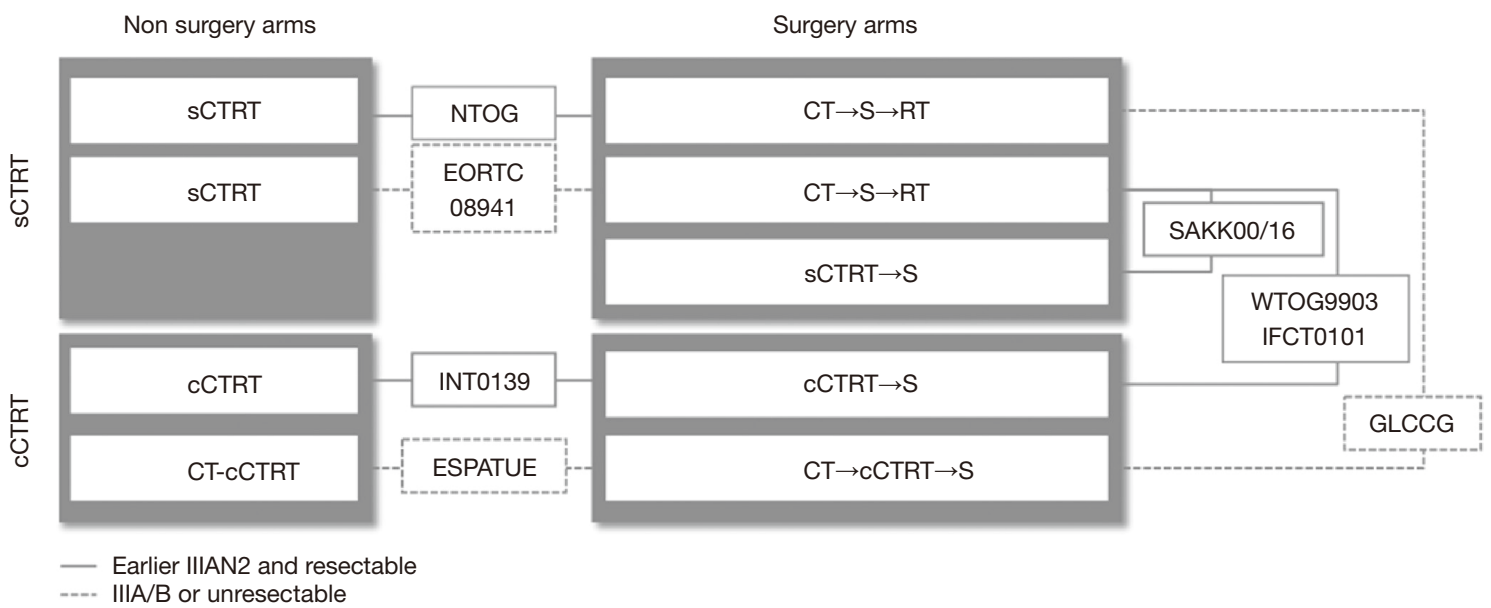

Figure 1 Design of most relevant clinical trials on induction therapy in stage III NSCLC. NSCLC, non-small cell lung cancer; CT, chemotherapy; cCTRT, concurrent chemoradiotherapy; RT, radiotherapy; sCTRT, sequential chemoradiotherapy; S, surgery.

chemoradiotherapy (CTRT) followed by durvalumab. Concomitant CTRT demonstrated superiority to sequential treatment in patients with stage III disease in terms of overall survival (OS) and reduced risk of locoregional progression (1). However, only $20-25 \%$ of patients with stage III NSCLC treated with concurrent CTRT will become long-term survivors $(1,2)$.

Several studies have attempted to improve outcomes in this clinical setting exploring novel systemic therapies such as cytotoxic compounds (pemetrexed), biological drugs (cetuximab or gefitinib), vaccines targeting specific glycoproteins (tecemotide) or escalating the dose of radiotherapy (3-6). After many negative clinical trials in this setting, in the PACIFIC trial consolidation therapy with durvalumab after concurrent CTRT improved diseasefree survival (DFS) and OS compared with placebo; thus, becoming the current standard of care in patients with locally advanced, unresectable, stage III NSCLC (7).

Clinical trials evaluating concurrent CTRT show that about one-third of the patients (35-43\%) will develop locoregional failure (8-10). Therefore, incorporating surgical resection to the treatment regimen of patients with stage III NSCLC may reduce the locoregional failure and improve the cure rate. Furthermore, surgical resection allows to examine treatment pathological response and tumor downstaging more accurately compared with radiological assessment. Induction therapy allows to start systemic treatment earlier and may increase compliance and tolerability of systemic therapy compared with adjuvant treatment. In this regard, the NSCLC Meta-analysis
Collaborative Group demonstrated that preoperative chemotherapy achieved comparable survival benefit to adjuvant chemotherapy in patients with stage II-III disease (11).

However, within this heterogeneous group of patients with locally advanced NSCLC, only a minority of patients with central tumors or enlarged, discrete hilar or mediastinal lymph nodes are potentially resectable. These patients should be considered for invasive mediastinal staging, evaluated by an experienced multidisciplinary team, and should receive multimodal treatment as standard of care.

In this review, we aim to analyze the lessons learned from clinical trials evaluating induction chemotherapy with or without radiotherapy followed by surgery in patients with stage III NSCLC and apply them to the growing evidence supporting the use of immunotherapy in the neoadjuvant setting.

\section{Role of surgery in stage IIIA-N2 NSCLC}

Four randomized controlled trials (RCTs) addressed the role of surgery in stage III NSCLC (Figure 1, Table 1). Two studies compared induction chemotherapy followed by surgery (with or without postoperative radiotherapy) versus definitive sequential CTRT [NTOG (12), EORTC08941 (13)] and two compared concomitant CTRT with or without surgery [INT0139 (14), ESPATUE (15)].

\section{NTOG trial (12)}

This phase III clinical trial randomized patients with IIIA- 


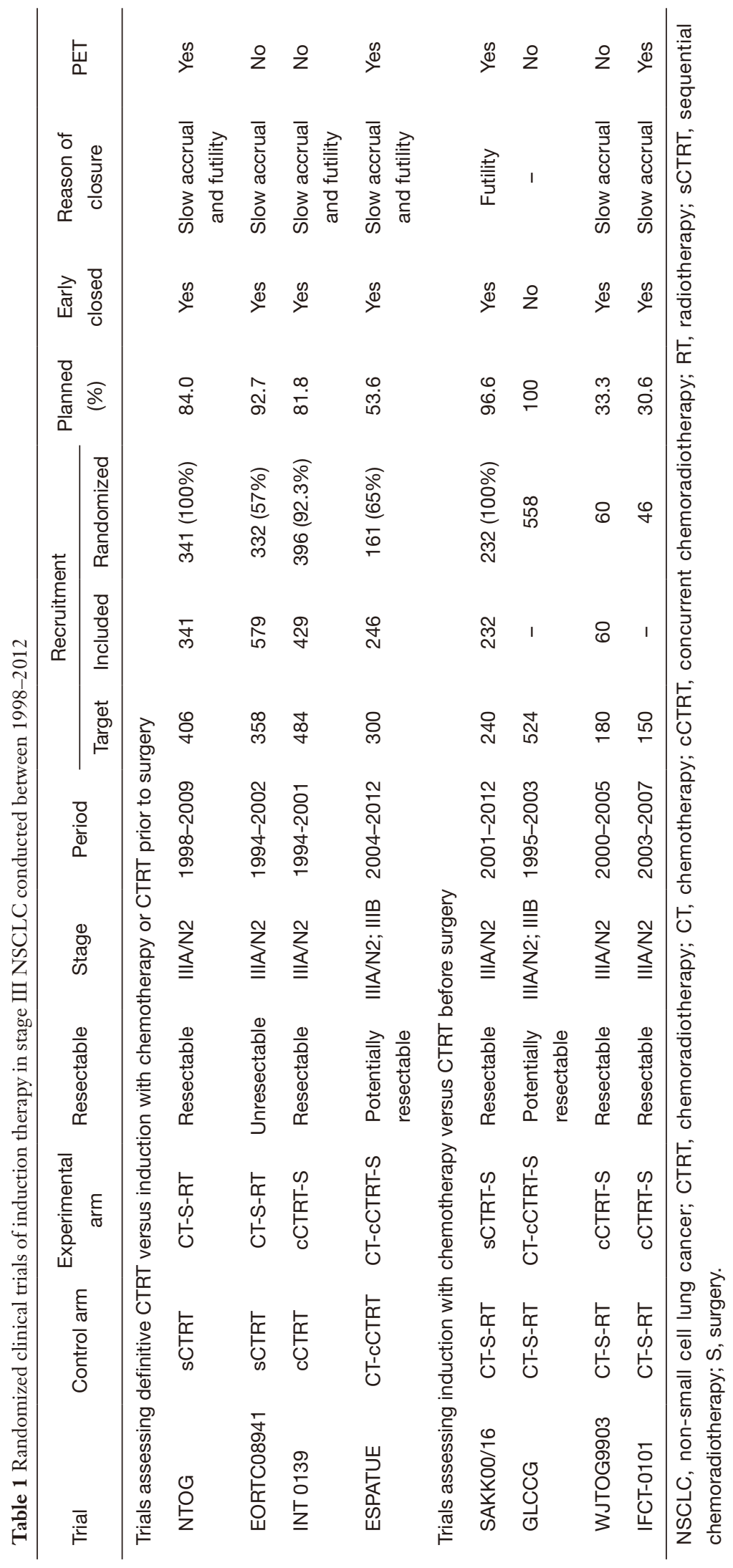


N2 NSCLC to induction with paclitaxel $225 \mathrm{mg} / \mathrm{m}^{2}$ plus carboplatin AUC6 every 3 weeks for 3 courses, followed by surgery and then postoperative radiotherapy ( $\operatorname{arm~A}$ ) versus definitive sequential CTRT (arm B). To detect an absolute increase of $10 \%$ in 5-year OS, 406 patients were estimated to be required. However, the study closed after recruiting 341 patients, since concomitant CTRT became standard of care instead of sequential treatment. Surgery was performed in 132 of 170 patients (78\%) and 121 (71\%) had a complete resection. Pathologic complete response (pCR) was achieved in $4 \%$ of patients. Median progression-free survival (PFS), OS and 5 -year survival rate were numerically longer in arm A (10 months, 17 months, and 20\%, respectively) compared with arm B (8 months, 15 months, and 16\%, respectively); however, these differences were not statistically significant. Despite carrying out this trial over 11 years in more than 300 patients, it was not sufficiently powered to detect a clinically relevant advantage of surgery after sequential CTRT.

\section{EORTC-08941 trial (13)}

This study addressed the same question as the NTOG trial but focused on stage IIIA-N2 patients deemed unresectable at baseline. The primary endpoint was OS and 640 patients were estimated to be needed to detect an absolute increase of $10 \%$ in 5 -year OS, with $80 \%$ power and type 1 -error of $5 \%$. Cisplatin-based induction chemotherapy was administered to 579 patients recruited over 8 years. Only 332 responding patients (57\%) were randomized to either surgery (with a $47 \%$ rate of pneumonectomy) or sequential radiotherapy (60 Gy in 30 fractions). In the surgery arm, only half of the 332 patients achieved complete resection, with persisting ypN2 disease in $57 \%$ of the patients. The rate of pCR was low (5\%) and there was no significant difference in OS (5-year OS was approximately $15 \%$ in both groups). A high mortality rate $(7 \%)$ was observed after pneumonectomy, leading to an extremely low longterm OS compared with other lung resections (5\% vs. 27\% of patients attaining 5-year OS, respectively). Overall, no survival benefit was observed from undergoing surgery after induction chemotherapy in patients with stage IIIA$\mathrm{N} 2$ disease that was unresectable at baseline and who were staged without using a PET scan.

\section{INT 0139 trial (14)}

This North American Intergroup clinical trial assessed the role of surgery following induction with CTRT. Patients with resectable stage IIIA-pN2 NSCLC received two cycles of cisplatin-etoposide concurrently with radiotherapy at a dose of 45 Gy in 25 fractions and were randomized to either surgery or completion of full dose radiotherapy (61 Gy). OS was the primary endpoint and, assuming a 25\% 2-year OS rate for the control arm, 484 patients were needed to detect a $10 \%$ absolute improvement in the surgical arm. The trial was closed prematurely after 7 years due to slow accrual and futility, with $82 \%$ of the sample size recruited. No significant differences in survival were observed between both arms (the 5 -year OS in the surgery arm was $27 \%$ vs. $20 \%$ in the non-surgery group; $\mathrm{P}=0.10$ ). A strikingly high mortality after pneumonectomy (25\%) was observed, with 14 of 16 treatment-related deaths occurring in the surgical group compared with only $3(2 \%)$ in the non-surgical group. In an adhoc exploratory analysis, there was an improvement in OS for patients who underwent lobectomy compared with a matched cohort of patients treated with chemoradiation, but not for those who underwent pneumonectomy.

\section{ESPATUE trial (15)}

This trial addressed the use of an intensive regimen in a population with a more advanced disease. Patients with stage IIIA-pN2 or highly selected, potentially resectable stage IIIB disease received induction chemotherapy (3 cycles of cisplatin plus paclitaxel) followed by hyperfractionated radiotherapy (45 Gy) given concurrently with cisplatin plus vinorelbine. Only responding patients deemed resectable by a tumor board were randomized to either surgery or definitive radiotherapy boost with 20-25 Gy (up to a total dose of 65-71 Gy). Three hundred patients were estimated to be needed to demonstrate an increase in 5-year OS from $25 \%$ to $40 \%$ with surgery. The trial started in 2004 and used contemporary staging methods such as brain imaging and 18F-FDG PET-CT and was closed in 2012, after an interim futility analysis when 246 patients had enrolled and 161 patients had been randomized (56\% of planned accrual). Among 81 patients allocated to surgery, 70 underwent surgery ( $25 \%$ had a pneumonectomy) and 66 achieved a complete resection, with a pCR rate of $33 \%(27 / 81)$. Mediastinal downstaging was not reported. Intriguingly, none of the treatment-related deaths in the surgery arm (5/70) were after pneumonectomy, in contrast with what was observed in other trials in this setting. The trial was negative at the futility analysis because the control arm performed as well as the experimental arm, with 
an impressive 5 -year OS of $40 \%$ and $44 \%$, respectively; the highest reported in this setting. Stage migration and selection bias after an intensive induction regimen might partially explain these outcomes.

Over two decades, more than 1,200 patients were treated in randomized trials; however, it was not possible to demonstrate a significant benefit in OS with surgery after induction chemotherapy or CTRT compared with definitive sequential or concomitant CTRT. Nevertheless, these trials were not powered to detect small but clinically relevant differences in OS. Moreover, achieving a complete resection, mediastinal downstaging, and $\mathrm{pCR}$ have emerged as prognostic factors of longer OS following induction treatment.

\section{Induction with chemotherapy versus CTRT before surgery in stage IIIA-N2 NSCLC}

Despite the discouraging results of trials comparing surgery versus thoracic radiotherapy after induction with chemotherapy or CTRT, additional clinical trials were designed to evaluate the role of chemotherapy alone versus CTRT before surgical resection in patients with locally advanced NSCLC (Figure 1, Table 1).

\section{SAKK00/16 trial (16)}

This is the only study comparing induction chemotherapy with or without sequential radiotherapy. Patients with stage IIIA-pN2 disease were randomized to induction treatment either with chemotherapy alone (cisplatin plus docetaxel) or with sequential CTRT (44 Gy in 22 fractions in 3 weeks), followed by surgery. To detect an improvement in event-free survival (EFS) of 6 months it was estimated that 240 patients would be required. After 11 years, the trial closed due to slow accrual, having almost completed the planned recruitment (232 patients). Although the rates of response (61\% vs. $44 \%$ ), complete resection ( $91 \%$ vs. $81 \%$ ), mediastinal downstaging (64\% vs. $53 \%)$ and pCR (16\% vs. $12 \%)$ were higher in patients treated with induction CTRT, there were no statistically significant differences either in EFS (12.8 vs. 10.5 months) or in OS (37.1 vs. 26.2 months). A limitation of this study was that thoracic radiotherapy was given sequentially instead of concurrently with chemotherapy.

\section{GLCCG trial (17)}

This study was sponsored by the German Lung
Cancer Cooperative Group (GLCCG) and assessed whether preoperative CTRT was superior to induction chemotherapy prior to surgery in patients with locally advanced NSCLC. Eligible patients had stage IIIA-N2 $(30 \%)$ or stage IIIB $(70 \%)$ that was deemed potentially resectable; although, they would've been considered unresectable in clinical practice. Patients were randomized to receive an induction regimen of 3 cycles of cisplatin plus etoposide alone or followed by twice daily radiation with concurrent carboplatin and vindesine. The primary endpoint was PFS and 500 patients were estimated to detect a 3 -month increase in OS from a median of 10 months in the control group. It took 8 years to complete the recruitment of 524 patients with stage III disease. Across both arms, there was a similar proportion of patients who underwent surgery. However, $44 \%$ of the patients did not undergo resection, given the high proportion of patients with stage IIIB that was enrolled. The rate of pneumonectomy was similar in both arms (35\%). As in the SAKK trial, addition of CTRT to induction showed higher rates of complete resection (55\% vs. 69\%), mediastinal downstaging (29\% vs. 46\%), and major pathologic response (MPR, 20\% vs. 60\%), which did not translate into a significant improvement in PFS or OS (median OS 17.6 vs. 15.7 months). Treatment-related deaths were higher in the interventional group compared with the control group, particularly after pneumonectomy (14\% vs. 6\%) but also after lobectomy (7.5\% vs. $2 \%)$.

Additional randomized clinical trials comparing different induction regimens were conducted in this clinical setting but closed early due to slow accrual.

\section{W7TOG9903 trial (18)}

This study was sponsored by West Japan Thoracic Oncology Group (WJTOG) and compared chemotherapy (carboplatin plus docetaxel) alone with chemotherapy plus 40 Gy of concurrent thoracic radiotherapy in patients with stage IIIA-N2. The trial was closed early after recruitment of only 60 of the 180 required patients. The addition of radiotherapy to induction chemotherapy conferred better local control but differences observed in OS were not significant due to lack of statistical power. Mediastinal downstaging was associated with better outcome after surgery.

\section{IFCT-0101 trial (19)}

This multicenter randomized phase II clinical trial was 
sponsored by the French Cooperative Thoracic Intergroup (IFCT). Patients with stage IIIA/pN2 tumors were randomized into 3 arms: chemotherapy alone with cisplatin plus gemcitabine or radiotherapy concurrent with either cisplatin plus vinorelbine or carboplatin plus paclitaxel. About 50 patients in each arm were needed to demonstrate the primary endpoint of feasibility, but the trial closed early due to very slow accrual after enrolling only 46 patients over 4 years. Amongst the 42 patients with resected tumors, 35 patients had a complete resection, 16 patients showed mediastinal downstaging, and $2(5 \%)$ patients achieved pCR. Feasibility was superior in the chemotherapy alone $\operatorname{arm}(93 \%)$ but the study was not powered to detect meaningful differences.

A systematic review and meta-analysis of 12 studies with 2,724 patients demonstrated higher tumor downstaging ( $\mathrm{P}=0.01)$, pCR $(\mathrm{P}=0.028)$, and local control $(\mathrm{P}=0.002)$ with preoperative CTRT compared with chemotherapy alone; however, it did not translate into a higher 5-year OS rate or a PFS benefit (20). Real world data has supported these findings. A study based on the SEER database showed that induction with CTRT led to a higher rate of mediastinal downstaging than chemotherapy alone in a cohort of T1-2N2 patients. However, induction with CTRT did not improve OS compared with chemotherapy alone (5-year OS of $41.4 \%$ vs. $40.8 \%$, respectively) (21).

Based on the abovementioned results from clinical trials, clinical guidelines for locally advanced NSCLC with discrete N2 involvement identified preoperatively recommend either definitive CTRT or induction with chemotherapy or CTRT followed by surgery $(22,23)$. Each treatment plan should be established within a multidisciplinary team with at least a thoracic surgeon, a medical oncologist, and a radiation oncologist. As preoperative chemotherapy is the preferred option rather than concurrent CTRT for resectable stage IIIA-N2 NSCLC, induction chemotherapy has been considered the control arm or the backbone in recent randomized clinical trials testing neoadjuvant immunotherapy.

\section{Clinical endpoints in trials evaluating neoadjuvant therapies}

OS and DFS are considered gold-standard endpoints in phase III clinical trials with curative intent in early-stage NSCLC. However, these long-term endpoints can take years to mature. To accelerate and increase the efficiency of clinical trials, surrogate endpoints reflecting the study intervention are often used to predict clinical benefit.

pCR, defined as the eradication of all tumor from resected primary tumor and lymph nodes, has been used as a surrogate endpoint of OS and DFS in breast cancer neoadjuvant clinical trials. However, its rarity in NSCLC ( $4 \%$, range, $0-16 \%$ ) has restricted its use as a surrogate in this clinical setting. MPR, defined as $\leq 10 \%$ of residual viable tumor (RVT), has been proposed as an alternate surrogate since it correlates with OS and DFS in patients with resected NSCLC after neoadjuvant chemotherapy, even after adjusting for pathological stage. MPR occurs in about $20 \%$ of patients with NSCLC after neoadjuvant chemotherapy and may predict long-term OS, but prospective studies are still needed to confirm its validity and reproducibility in this setting (24). As these surrogate endpoints were developed in the context of neoadjuvant chemotherapy, its validity after novel therapies such as immunotherapy is unknown and should be investigated. Due to the histological changes seen in the regression bed (dense immune infiltration by lymphocytes, macrophages and tertiary lymphoid structures; massive tumor cellcholesterol clefts; tissue repair-neovascularization and proliferative fibrosis), an immune-related RVT (irRVT) has been proposed with improved interobserver consistency compared to classic RVT (25). This novel endpoint should be prospectively evaluated in trials assessing immunotherapy alone or in combination with other therapies to confirm its ability to predict DFS and OS.

\section{Immune checkpoint inhibitors (ICIs) as neoadjuvant treatment in resectable stage III NSCLC}

ICIs against the programmed death-1 (PD-1) and programmed death-ligand 1 (PD-L1) have transformed treatment for patients with locally advanced or metastatic NSCLC. Significant improvements in OS have established ICIs as standard of care in the first-line setting in advanced NSCLC and as consolidative treatment after concurrent CTRT in stage III NSCLC (26). The combination of ICI with chemotherapy also improved results in phase III clinical trials (27-33) and appears to successfully induce immunogenic cell-death and inhibit the immunosuppressive tumor microenvironment $(34,35)$.

The immune cell composition changes as the tumor progresses: earlier stages are enriched for T-effector memory CD8+ cells, while advanced tumors are enriched for Tregs and myeloid-derived suppressive cells (36). 


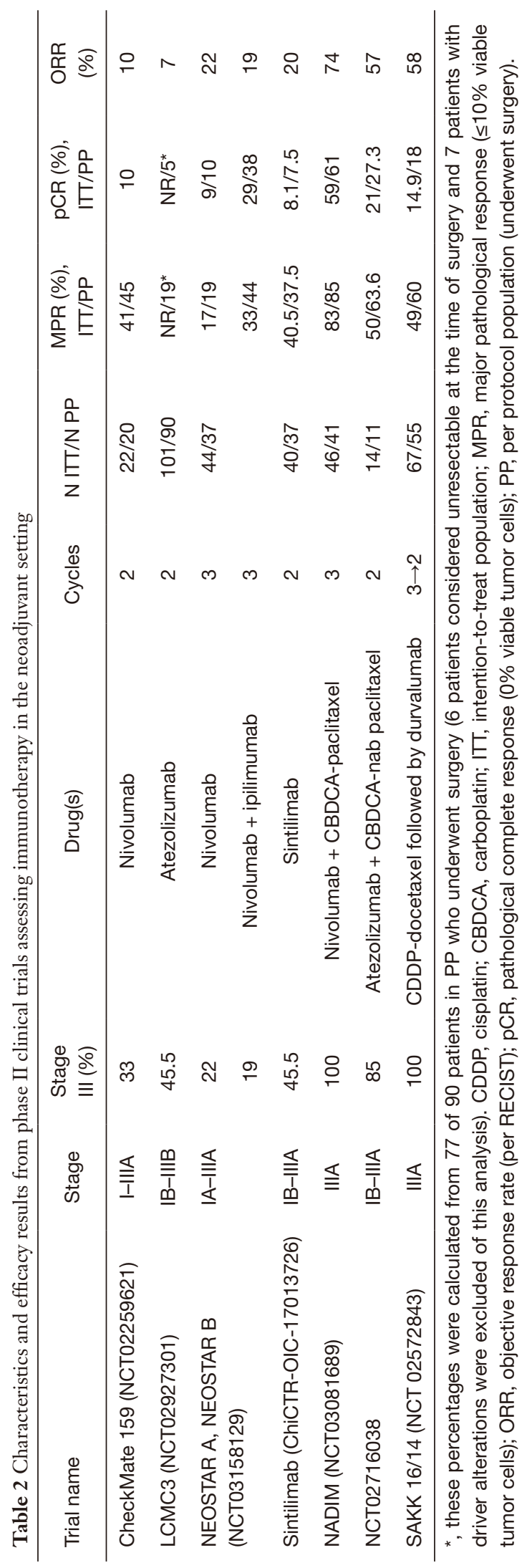

Preclinical and clinical studies have suggested that neoadjuvant ICI might be superior to adjuvant treatment because it can induce a stronger and more prolonged systemic and antitumor T-cell immune response (37-39). Intact primary tumor and drainage to the regional lymph nodes are thought to induce greater immune response compared with the micro-metastatic disease remaining after surgical resection of the tumor. Several studies have explored the activity of ICI alone or in combination with chemotherapy to improve the cure rate in patients with earlier stages of NSCLC.

\section{Clinical trials evaluating ICI or ICI plus chemotherapy in the neoadjuvant setting}

We have reviewed the studies that assessed the safety and efficacy of induction with anti-PD-1 or anti-PD-L1 therapies given alone or in combination with anti-CTLA-4 or platinum-based chemotherapy. All studies are listed in Table 2.

\section{CheckMate 159 trial (40)}

This single arm, phase Ib-II, clinical trial is the seminal study assessing neoadjuvant anti-PD-1 therapy in patients with resectable stage IB-IIIA NSCLC (40). Two doses of nivolumab were administered prior to surgery in 22 patients. The primary endpoints were safety and feasibility and the key secondary were radiologic and pathological responses. Neoadjuvant nivolumab showed an acceptable safety profile and there was only one grade $\geq 3$ immune-related adverse event (irAE) (skin rash). Twenty of the 22 patients enrolled underwent resection (one patient was deemed unresectable and other had small cell carcinoma histology). Surgery was not delayed in any patient and there was no operative mortality (a single patient died of traumatic injury on postoperative day 61). Morbidity occurred in 10 of 20 patients $(50 \%)$. The most common postoperative complication was atrial arrhythmia $(6 / 20 ; 30 \%)$. The other complications were a concomitant myocardial infarction, pneumonia and pulmonary embolism, prolonged air leak and urinary infection, and empyema (41). Of the 21 patients evaluable for radiographic response, 2 patients (10\%) had a partial response (PR), 18 (86\%) had stable disease (SD), and $1(5 \%)$ had disease progression (PD). MPR evaluated in the tumor and lymph nodes was observed in $45 \%$ (95\% CI: $23-68 \%)(9 / 20)$ of resected tumors and 2 patients achieved pCR. Pathological responses occurred irrespective of PD- 
L1 expression but were correlated with the pretreatment tumor mutational burden (TMB). After a median follow up of 34.6 months, median recurrence-free survival (RFS) and OS had not been reached yet.

\section{NEOSTAR trial (42)}

This is a non-comparative phase II clinical trial testing nivolumab alone or in combination with ipilimumab prior to surgery in patients with stage I-IIIA resectable NSCLC. The study enrolled 44 patients who were randomized to neoadjuvant nivolumab $(3 \mathrm{mg} / \mathrm{kg}$ on days $1,15,29)(\mathrm{n}=23)$ or nivolumab plus ipilimumab $(1 \mathrm{mg} / \mathrm{kg}$ on day 1$)(\mathrm{n}=21)$. The primary endpoint was MPR. Across both arms, 39 patients underwent post-ICI surgery; however, 2 patients were resected off trial because they received chemotherapy in addition to ICI (1 patient with suspected PD and 1 patient with grade 3 immune-related diarrhea). Five patients did not undergo surgery due to: 2 patients with PD, 1 patient with grade 3 hypoxia from pleural effusion, 1 patient had high surgical risk, and 1 patient declined surgery (42). The overall response rate (ORR) by RECIST v1.1 was $20 \%$ [ 8 PR (5 nivolumab, 3 combination) and 1 CR (combination)] and $14 \%$ of patients had PD (3 nivolumab, 3 combination). Among the intention-to-treat (ITT) population $(n=44)$, 11 patients achieved MPR (25\%), 4 in the nivolumab arm and 7 in the combination arm. Eight patients achieved pCR (15\%), 2 in the nivolumab arm and 6 in the combination arm. Among the 37 patients with on-study resection, the MPR rate was 30\% (4 receiving nivolumab, 7 receiving the combination). Objective response by RECIST was higher in patients who achieved MPR than in those that did not [7 (78\%) vs. 2 (22\%); $\mathrm{P}<0.001]$. A novel phenomenon, called nodal immune flare (NIF), was observed in some patients who experienced apparent radiographic nodal progression, but the pathologic examination revealed only granulomas without the presence of tumor cells. There were 8 patients $(22 \%)$ in whom surgery was delayed beyond 42 days ( 3 patients in the nivolumab arm and 5 in the combination arm). Surgical complications included 2 bronchopleural fistulas in the nivolumab arm and 8 prolonged air leaks ( 5 in patients receiving nivolumab, 3 receiving the combination). Grade $\geq 3$ treatment-related adverse events (TRAEs) included a death due to bronchopleural fistula following an immune-related pneumonitis treated with corticosteroids (grade 5 in a patient receiving nivolumab); grade 3 pneumonia, hypoxia, hypermagnesemia (1 each, all receiving nivolumab) and grade 3 diarrhea, hyponatremia
(1 each, all receiving the combination). Baseline tumor PD$\mathrm{L} 1$ expression was associated with radiological $(\mathrm{P}=0.015)$ and pathological responses $(\mathrm{P}=0.015)(43)$.

\section{LCMC3 trial (44)}

In this single arm phase II clinical trial, patients with resectable stage IB-IIIB NSCLC received neoadjuvant atezolizumab (1,200 $\mathrm{mg}$ for 2 cycles) followed by surgery and then standard of care chemotherapy followed by atezolizumab for 1 year. The interim analysis was based on the first 101 of 180 planned patients. Stage IIIA and IIIB accounted for $39 \%$ and $7 \%$ of the patients, respectively. The primary endpoint was the MPR rate. Of 101 patients, 11 patients were not resected due to: 5 patients with $\mathrm{PD}$, 4 patients withdrew informed consent, 1 patient had echocardiogram alterations, and 1 patient was deemed unresectable. The primary efficacy population $(n=77)$ excluded 6 patients without MPR assessment and 7 patients who harbored driver mutations. The MPR rate in the primary population was $19 \%(15 / 77,95 \%$ CI: $11-30)$ and 4 patients $(5 \%)$ had pCR. In per protocol (PP) population $(\mathrm{n}=90), 6$ patients $(7 \%)$ had PR, $80(89 \%)$ had SD and $4(4 \%)$ had PD by RECIST v1.1. The 12-month DFS calculated from the date of surgery was $89 \%$ (95\% CI: not reported). Pre-operative irAEs were reported in 30 patients (30\%). The majority of them were grade 1 or 2 (12 skin rash, 10 infusion-related reaction, 4 abnormal thyroid function and 5 elevated liver enzymes) and only 1 grade 3 was reported (pneumonitis). In this study, pathological regression and MPR did not correlate with PD-L1 expression or with TMB evaluated by whole exome sequencing.

\section{ChiCTR-OIC-17013726 trial (45)}

This single arm phase II trial assessed the efficacy and safety of sintilimab, an anti-PD-1 antibody, in the neoadjuvant setting in patients with resectable stage IB-IIIA NSCLC. Primary endpoints were drug safety, surgical complications, and no-delay of surgery rate. Secondary endpoints were ORR, MPR, DFS at 1 and 2 years and OS at 2 years. Forty patients received 2 cycles of sintilimab (200 $\mathrm{mg}$ IV on days 1 and 22) of whom 37 underwent surgery. Two patients had a treatment-related operation delay. Most patients received adjuvant sintilimab alone $(40.5 \%)$, but some patients received adjuvant chemotherapy plus sintilimab (27\%) or conventional adjuvant chemotherapy (11\%). The ORR was $20 \%$ and 15 patients achieved MPR (40.5\%, 95\% CI: $24.8-$ 
$57.9 \%)$, including 6 who had pCR (16.2\%, 95\% CI: $6.2-$ $32.0 \%)$. Twenty-one patients (52.5\%) had TRAEs during the neoadjuvant phase, but only 4 patients (10\%) reported grade 3-4 TRAEs and one patient had a grade 5 TRAE.

\section{NADIM trial (46)}

This single arm phase II clinical trial was the first multicenter study testing chemo-immunotherapy in the neoadjuvant setting in patients with resectable stage IIIA NSCLC. This academic study was sponsored by the Spanish Lung Cancer Group (GECP). Neoadjuvant treatment consisted of three cycles of nivolumab $360 \mathrm{mg}$ + paclitaxel $200 \mathrm{mg} / \mathrm{m}^{2}$ + carboplatin AUC 6 IV Q3W prior to surgery. Adjuvant treatment with nivolumab was given for 1 year (nivolumab $240 \mathrm{mg}$ IV Q2W for 4 months and then nivolumab $480 \mathrm{mg}$ IV Q4W for 8 months). The primary endpoint was PFS at 24 months from the first dose of neoadjuvant treatment. Forty-six patients were included and received neoadjuvant treatment. In the ITT population $(\mathrm{n}=46), 34$ patients (74\%, 95\% CI: 60-85\%) achieved MPR and 24 patients had pCR (52\%, 95\% CI: 38-66\%). Based on RECIST v1.1, the ORR was $74 \%$ (69.6\% and $4.3 \%$ achieved PR and CR, respectively). The neoadjuvant treatment was generally well tolerated, and there were no surgery delays. All surgical resections were R0 and no intraoperative complications were seen. Only 12 patients (29\%) presented postsurgical complications; the most frequent were: 5 patients with respiratory infections, 4 patients with episodes of cardiac arrhythmias, and 2 patients with prolonged air leak. There was no surgeryrelated postoperative mortality. After a median follow-up of 17.1 months, PD was reported in 6 patients (1 patient with a CR but found to harbor an EGFR mutation, 3 patients with $>10 \%$ of RVT, and 2 patients with no surgery). In the PP population ( $\mathrm{n}=41$ ), the MPR was $83 \%$ (95\% CI: $68-93 \%)$ and pCR 59\% (95\% CI: 42-74\%). Seven patients (17\%) patients presented $>10 \%$ of RVT. Within the overall ITT population, the PFS at 12 and 18 months was 96\% (95\% CI: $84-99 \%)$ and $81 \%$ (95\% CI: 61-91\%), respectively. The OS rate at 12 and 18 months was $98 \%$ (95\% CI: 85$100 \%$ ) and $91 \%$ (95\% CI: 73-97\%). In the PP population, the PFS rate at 12 and 18 months was $100 \%$ and $87 \%$ (95\% CI: 64-96\%), respectively and the OS rate at 12 and 18 months was $100 \%$ and $95 \%$ (95\% CI: 68-99\%). In terms of safety, $24.2 \%$ of the patients reported grade $\geq 3$ TRAEs. The majority of these TRAEs were chemotherapy-related and only one patient reported a grade 3 TRAE (nephritis).

\section{NCT02716038 trial (47)}

A single-arm phase II clinical trial evaluated the combination of atezolizumab with carboplatin plus nab-paclitaxel in patients with resectable stage IB-IIIA NSCLC. The number of induction cycles administered in this study was based on the radiological response after the first two cycles. Patients with a response could receive two additional cycles, while patients in whom tumor progression was observed went directly to surgery. The primary endpoints were MPR and time to surgery. Fourteen evaluable patients (85\% stage IIIA) were enrolled. Eleven patients underwent resection successfully and 7/14 (50\%) patients achieved MPR, including 3 patients (21\%) with pCR. Pathological responses were observed regardless of PD-L1 expression. Eight patients (57\%) had radiologic PR and the remainder had SD. With a median follow-up of 8.6 months, PD was reported in 4 patients ( 2 chest recurrences and 2 brain metastases). The most common toxicity was neutropenia (71\% grade $3-4)$, with $64 \%$ patients requiring a dose reduction of chemotherapy.

\section{SAKK16/14 (48)}

A single-arm phase II clinical trial evaluated three cycles of cisplatin plus docetaxel followed by two cycles of durvalumab in patients with resectable stage IIIA NSCLC. The primary endpoint was EFS. Sixty-seven evaluable patients were enrolled, but only 55 patients underwent resection. Thirty-three patients achieved MPR, including 10 patients with pCR. Thirty-six out of 62 (58\%) patients had radiologic response. With a median follow-up of 28 months, 14 patients had progressed and median EFS has not been reached. The EFS at 1 year was $73.3 \%$ (95\% CI: 62.5-81.4). There were two fatal adverse events (AEs): one respiratory failure during the induction chemotherapy and one bronchopulmonary hemorrhage after surgery.

There are several ongoing phase II/III clinical trials assessing ICI in combination with chemotherapy in the neoadjuvant setting (Table 3).

NADIM II (NCT03838159) is an open-label randomized phase II clinical trial sponsored by the Spanish Lung Cancer Group. Ninety patients will be enrolled to determine the pCR in patients treated with the NADIM neoadjuvant chemo-immunotherapy schema followed by a shorter adjuvant treatment with nivolumab for 6 months versus carboplatin plus paclitaxel for three cycles followed by surgery. 


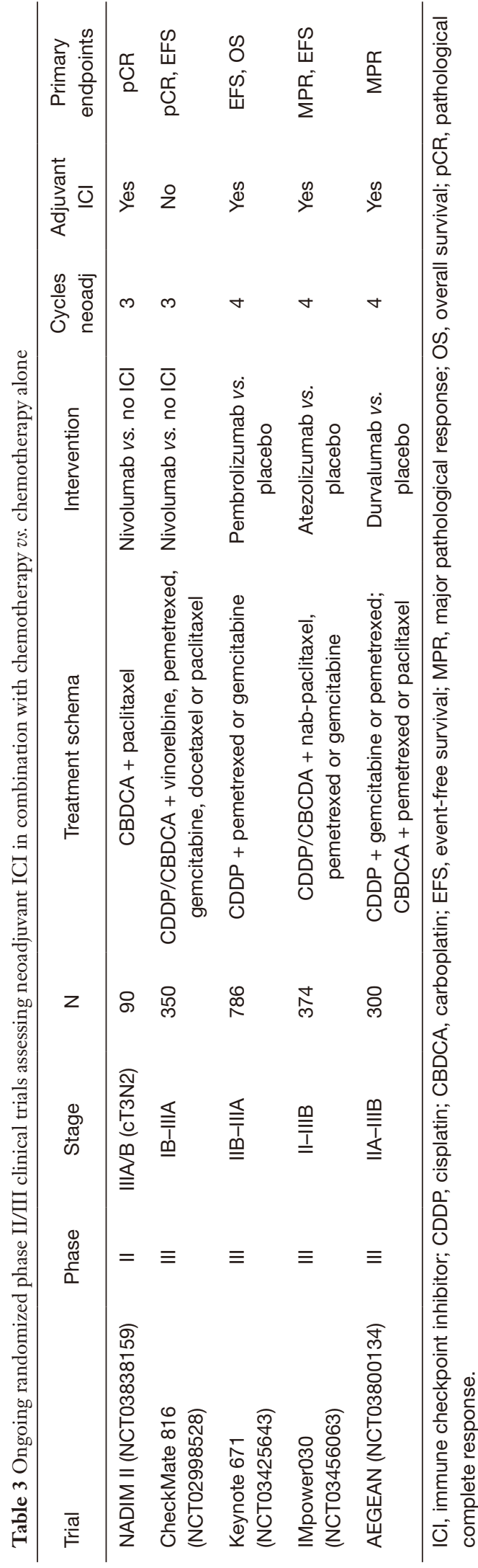

CheckMate 816 (NCT02998528) is an international, open-label, randomized phase III clinical trial in patients with stage IB-IIIA NSCLC comparing neoadjuvant treatment with nivolumab plus platinum-based chemotherapy versus platinum-based chemotherapy alone. This study originally had a third arm with ipilimumab plus nivolumab that was stopped. Co-primary endpoints are EFS and $\mathrm{pCR}$ rate.

KEYNOTE-671 (NCT03425643) is an international, double-blinded, randomized phase III clinical trial investigating the combination of neoadjuvant platinum-based chemotherapy plus placebo prior to surgery and adjuvant placebo for 1 year versus neoadjuvant platinum-based chemotherapy plus pembrolizumab prior to surgery and adjuvant pembrolizumab in patients with resectable stage IIB or IIIA NSCLC. Primary endpoints are EFS and OS.

IMpower030 (NCT03456063) is a multicenter, doubleblinded, randomized phase III clinical trial assessing atezolizumab efficacy in combination with platinumbased chemotherapy in patients with resectable (II, IIIA or selected IIIB) NSCLC. Patients received four cycles of atezolizumab versus placebo combined with platinum-based chemotherapy. After surgery only those patients randomized to atezolizumab will continue ICI treatment until a maximum number of 16 cycles. Co-primary endpoints are MPR and EFS.

Durvalumab is also currently being investigated in several phase II neoadjuvant studies, including the IONESCO study (NCT03030131), NCT02572843 (durvalumab plus cisplatin + docetaxel) and NCT02904954 (durvalumab plus stereotactic body radiation therapy) and in the phase III clinical trial AEGEAN (NCT03800134) in combination with platinum-based chemotherapy.

\section{Biomarkers of response to ICI neoadjuvant therapy}

Neoadjuvant trials are an ideal setting for exploring predictive biomarkers. PD-L1 expression and TMB, biomarkers for immunotherapy in advanced NSCLC, have not shown a consistent association with response to neoadjuvant immunotherapy. Tumor responses occurred in both PD-L1 positive and PD-L1 negative tumors and whereas a positive correlation between the pathological response and the baseline $\mathrm{TMB}$ was reported in the LCMC3 trial (40), no correlation was found between both parameters.

In the seminal neoadjuvant trial of nivolumab 
(CheckMate 159), T-cell receptor (TCR) repertoire was significantly expanded in patients who achieved MPR after the second dose of nivolumab (49). In addition, clearance of plasma circulating tumor DNA (ctDNA) prior to surgery was detected in all patients who achieved $\geq 30 \%$ reduction in RVT and had pre-treatment detectable ctDNA. Furthermore, higher intratumoral TCR clonality was correlated with reduced residual tumor at the time of the surgery; peripheral expansion of tumor-specific T-cells and long-term persistence were associated with longer DFS (50).

In the NEOSTAR trial, the TCR repertoire was measured in pre-treatment biopsies, surgical specimens, matched adjacent normal lung, as well as paired blood at baseline, prior to surgery, and 8 weeks post-surgery $(n=44)$. Neoadjuvant therapy benefit was associated with higher clonality in tumors and lower clonality in blood posttherapy, suggesting increased $\mathrm{T}$ cell trafficking into the tumor (differences not statistically significant). Moreover, higher pretreatment TCR clonality in the blood was associated with a lower percentage of RVT at time of surgery in both treatment arms $(\mathrm{P}=0.04)$ (51).

In the atezolizumab with chemotherapy trial (NCT02716038), blood immune phenotyping by flow cytometry $(\mathrm{n}=61)$ showed that $\mathrm{T}$ - and NK-cells levels were lower at baseline in patients who achieved MPR than in those who did not achieve MPR. Among 48 patients with paired pre- and post-treatment peripheral blood samples, the expansion of NK-cells and granulocytes and the contraction of monocytes were significantly correlated with achieving a MPR. Furthermore, a lymph node immunephenotyping analysis performed in 28 patients showed a significant increase in memory T-cells $(\mathrm{CD} 3+\mathrm{CD} 27+$ $\mathrm{CD} 45 \mathrm{RO}+$ ) and a decrease in regulatory T-cells (CD3+ CD4+ CD25+) in the MPR population. The association between immune cells and irAEs was explored in 55 patients, showing that granulocytes and dendritic subsets were higher in patients who developed irAEs (44).

In the NADIM trial, blood samples were obtained preand post-neoadjuvant treatment. Absolute number of cells in the hemogram $(\mathrm{n}=41)$ and peripheral blood mononuclear immune cells (PBMCs) $(\mathrm{n}=27)$ were analyzed as potential biomarkers of response to immunotherapy. A higher decrease on the platelets-to-lymphocytes ratio (PLR) post treatment was associated with incomplete pathological response ( $\geq 10 \%$ RVT). Higher levels of PD-1 expression in CD4 $\mathrm{T}$ cells at baseline was associated with pCR. A reduction of PD-1 detection in CD4 T cells, CD8 T cells and NK cells and a decrease in CD4 T cells and NK cells activation after neoadjuvant treatment was also associated to pCR (52).

Due to the limited number of samples analyzed across these studies, the differences in the techniques employed, and the short follow-up of these clinical trials, further clinical trials are needed to validate biomarkers predicting benefit from neoadjuvant ICI.

\section{Discussion}

Locally advanced NSCLC is a highly heterogeneous disease requiring a multidisciplinary team-based treatment approach. As more than a third of the patients (35-43\%) will develop locoregional failure and only a quarter of the patients $(20-25 \%)$ will achieve long-term survival with concurrent CTRT, surgery has been intensively studied in patients with discrete N2 involvement and resectable disease. However, most trials evaluating surgery in stage III NSCLC following induction treatment were not powered to detect small differences in survival outcomes. Induction chemotherapy with radiotherapy was associated with higher rates of tumor downstaging and pCR than chemotherapy alone (4-5\% vs. 16-60\%, respectively); however, these did not translate into better long-term outcomes, nor higher cure rates (5-year OS $41.4 \% v s$. $40.8 \%$, respectively). Therefore, induction chemotherapy has been adopted as the control arm in clinical trials evaluating neoadjuvant immunotherapy. The percentage of RVT and MPR following neoadjuvant treatment has been evaluated or proposed as a surrogate marker for OS and DFS. As a limitation, those surrogate endpoints cannot capture TRAEs or whether lung resection has been feasible, which is highly relevant when novel agents are being tested for potentially curable disease. Furthermore, those endpoints should also be reinforced by long-term survival outcomes and be consistent with a higher cure rate. Several studies assessed the feasibility and efficacy of induction ICI alone or in combination with chemotherapy in early stage and resectable stage IIIA NSCLC. Neoadjuvant ICI monotherapy trials reported promising rates of MPR $(17-45.5 \%)$ as well as pCR (9-29\%), with relatively low toxicity and low progression rates without compromising resection rates (Figure 2). The addition of ICI to platinumbased chemotherapy reported an unprecedented MPR rate of $50-83 \%$ and pCR of $21-59 \%$ (Figure 2). If these encouraging efficacy and safety results are confirmed in currently ongoing randomized clinical trials, induction based on chemotherapy plus ICI followed by surgery could 


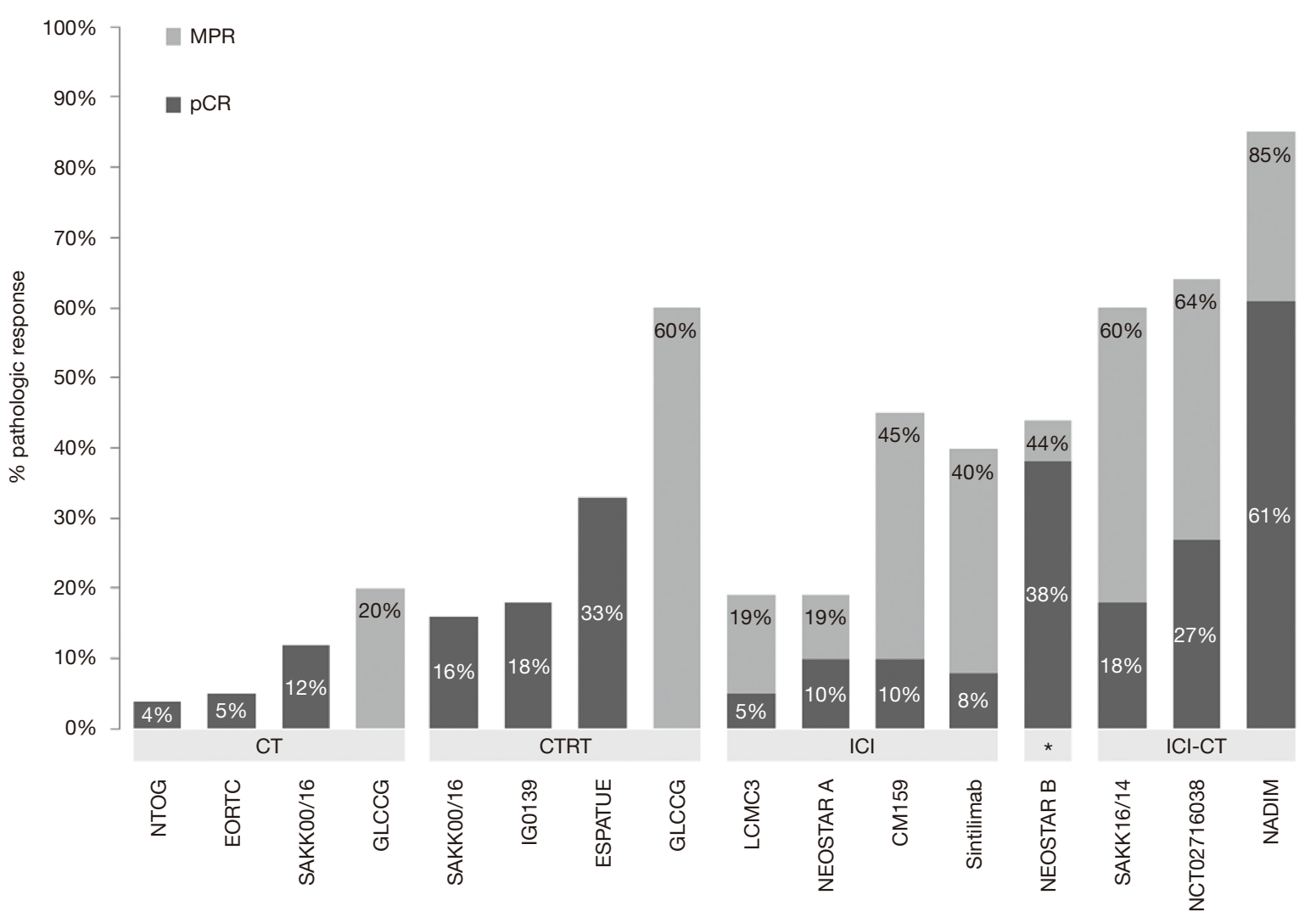

Figure 2 Rate of pathologic response, including pCR and MPR in clinical trials evaluating induction therapy in stage III NSCLC. *, ICI doublet. pCR, pathologic complete response; MPR, major pathologic response; NSCLC, non-small cell lung cancer; CT, chemotherapy; CTRT, chemoradiotherapy; ICI, immune checkpoint inhibitor.

become standard of care for patients with resectable stage III NSCLC. Induction CTRT could still be considered standard of care in tumors invading chest wall or potentially resectable $\mathrm{T} 4$, but the high rate of pathological response achieved with neoadjuvant chemotherapy combined with ICI warrants further research also in these clinical scenarios.

Incorporating immunotherapy to the preoperative setting has several challenges including the assessment of radiologic and pathologic response, which indeed can be discordant. In this regard, pseudoprogression or the flare phenomenon have been observed and might mistakenly consider patients as inoperable. Standardized approaches to determine the degree of pathologic response are needed. Unfortunately, novel endpoints, such as immune-related percentage of RVT (\% irRVT) are not prespecified endpoints in the ongoing clinical trials. Conversely, treatment strategies for those patients who will not achieve mediastinal downstaging or MPR have yet to be defined and the role of postoperative radiotherapy has not yet been established. Administering adjuvant treatment with ICI in the experimental arm is an additional limitation of ongoing clinical trials because it will not determine whether it is needed in all patients or only in those who do not achieve clinically relevant pathological response.

Identifying biomarkers for neoadjuvant ICI (alone or in combination with chemotherapy) is crucial to provide patients with the best therapeutic approaches and avoid induction therapy in patients at risk of progression and who may benefit from definitive concurrent CTRT. Although neoadjuvant trials provide an ideal platform for biomarker assessment, the association of PD-L1 and TMB with response to induction with immunotherapy has not been consistent in phase I/II clinical trials. This might be explained both due to differences in the study population or in the methods used, but also due to their limited statistical power for validating the predictive value of those markers.

The most appropriate treatment in patients with locally advanced NSCLC should be decided in the context of 
experienced multidisciplinary tumor boards with the participation of thoracic surgeons with expertise not only in lung cancer surgery following neoadjuvant chemotherapy or CTRT, but also following induction with ICI. Although we anticipate a paradigm shift in the preoperative treatment of patients with stage III resectable NSCLC, we should wait for the results from randomized clinical trials evaluating induction with chemotherapy plus ICI before incorporating this treatment regimen into the clinical practice. We consider that allowing patients to participate in ongoing clinical trials is currently the most suitable strategy in this clinical setting. These clinical trials might validate pathological response as a surrogate marker of survival benefit in this clinical setting and will probably confirm whether this therapeutic strategy can improve the cure rate in patients with stage II-III NSCLC.

\section{Acknowledgments}

Copyediting editorial support was provided by Aurora O'Brate.

Funding: NV is supported by a Rio Hortega scholarship (CM19/00245). EN received support from the SLT006/17/00127 grant, funded by the Department of Health of the Generalitat de Catalunya by the call "Acció instrumental d'intensificació de professionals de la salut". We thank CERCA Program/Generalitat de Catalunya for their institutional support and grant 2017SGR448.

\section{Footnote}

Provenance and Peer Review: This article was commissioned by the Guest Editor (Mariano Provencio) for the series "Multimodal management of locally advanced N2 nonsmall cell lung cancer" published in Translational Lung Cancer Research. The article has undergone external peer review.

Peer Review File: Available at http://dx.doi.org/10.21037/ tlcr-20-420

Conflicts of Interest: All authors have completed the ICMJE uniform disclosure form (available at http://dx.doi. org/10.21037/tlcr-20-420). The series "Multimodal management of locally advanced N2 non-small cell lung cancer" was commissioned by the editorial office without any funding or sponsorship. EN participated in advisory boards from Bristol Myers Squibb, Merck Sharpe \& Dohme,
Lilly, Roche, Pfizer, Takeda, Boehringer Ingelheim, Amgen and AstraZeneca; EN has participated as investigator of the NADIM, NADIM-II and Keynote-671 clinical trials and has received research funding from Roche and Pfizer. RP participated in advisory boards from Bristol Myers Squibb, Merck Sharpe \& Dohme, Lilly, Roche, Pfizer, Boehringer Ingelheim and AstraZeneca. NV reports personal fees and non-financial support from Roche, personal fees and nonfinancial support from Boehringer Ingelheim, personal fees from Bristol Mayers Squibb, personal fees and non-financial support from Pfizer, personal fees and non-financial support from Lilly, outside the submitted work. The authors have no other conflicts of interest to declare.

Ethical Statement: The authors are accountable for all aspects of the work in ensuring that questions related to the accuracy or integrity of any part of the work are appropriately investigated and resolved.

Open Access Statement: This is an Open Access article distributed in accordance with the Creative Commons Attribution-NonCommercial-NoDerivs 4.0 International License (CC BY-NC-ND 4.0), which permits the noncommercial replication and distribution of the article with the strict proviso that no changes or edits are made and the original work is properly cited (including links to both the formal publication through the relevant DOI and the license). See: https://creativecommons.org/licenses/by-nc-nd/4.0/.

\section{References}

1. Aupérin A, Le Péchoux C, Rolland E, et al. Meta-analysis of concomitant versus sequential radiochemotherapy in locally advanced non-small-cell lung cancer. J Clin Oncol 2010;28:2181-90.

2. O'Rourke N, Roqué I Figuls M, Farré Bernadó N, et al. Concurrent chemoradiotherapy in non-small cell lung cancer. Cochrane Database Syst Rev 2010;(6):CD002140.

3. Senan S, Brade A, Wang LH, et al. PROCLAIM: randomized phase III trial of pemetrexed-cisplatin or etoposide-cisplatin plus thoracic radiation therapy followed by consolidation chemotherapy in locally advanced nonsquamous non-small-cell lung cancer. J Clin Oncol 2016;34:953-62.

4. Kelly K, Chansky K, Gaspar LE, et al. Phase III trial of maintenance gefitinib or placebo after concurrent chemoradiotherapy and docetaxel consolidation in inoperable stage III non-small-cell lung cancer: SWOG 
S0023. J Clin Oncol 2008;26:2450-6.

5. Bradley JD, Hu C, Komaki RR, et al. Long-term results of NRG oncology RTOG 0617: standard- versus highdose chemoradiotherapy with or without cetuximab for unresectable stage III non-small-cell lung cancer. J Clin Oncol 2020;38:706-14.

6. Butts C, Socinski MA, Mitchell PL, et al. Tecemotide (L-BLP25) versus placebo after chemoradiotherapy for stage III non-small-cell lung cancer (START): a randomised, double-blind, phase 3 trial. Lancet Oncol 2014;15:59-68.

7. Antonia SJ, Villegas A, Daniel D, et al. Overall survival with durvalumab after chemoradiotherapy in stage III NSCLC. N Engl J Med 2018;379:2342-50.

8. Yamamoto N, Nakagawa K, Nishimura Y, et al. Phase III study comparing second- and third-generation regimens with concurrent thoracic radiotherapy in patients with unresectable stage III non-small-cell lung cancer: West Japan Thoracic Oncology Group WJTOG0105. J Clin Oncol 2010;28:3739-45.

9. Segawa Y, Kiura K, Takigawa N, et al. Phase III trial comparing docetaxel and cisplatin combination chemotherapy with mitomycin, vindesine, and cisplatin combination chemotherapy with concurrent thoracic radiotherapy in locally advanced non-small-cell lung cancer: OLCSG 0007. J Clin Oncol 2010;28:3299-306.

10. Curran WJ Jr, Paulus R, Langer CJ, et al. Sequential vs. concurrent chemoradiation for stage III non-small cell lung cancer: randomized phase III trial RTOG 9410. J Natl Cancer Inst 2011;103:1452-60.

11. NSCLC Meta-analysis Collaborative Group. Preoperative chemotherapy for non-small-cell lung cancer: a systematic review and meta-analysis of individual participant data. Lancet 2014;383:1561-71.

12. Sorensen JB, Ravn J, Pilegaard HK, et al. Surgery for NSCLC stages T1-3N2M0 having preoperative pathologically verified N2 involvement: a prospective randomized multinational phase III trial by the Nordic Thoracic Oncology Group. J Clin Oncol 2013;31:abstr 7504.

13. van Meerbeeck JP, Kramer GW, Van Schil PE, et al. Randomized controlled trial of resection versus radiotherapy after induction chemotherapy in stage IIIA-N2 non-small-cell lung cancer. J Natl Cancer Inst 2007;99:442-50.

14. Albain KS, Swann RS, Rusch VW, et al. Radiotherapy plus chemotherapy with or without surgical resection for stage III non-small-cell lung cancer: a phase III randomised controlled trial. Lancet 2009;374:379-86.
15. Eberhardt WE, Pottgen C, Gauler TC, et al. Phase III study of surgery versus definitive concurrent chemoradiotherapy boost in patients with resectable stage IIIA(N2) and selected IIIB non-small-cell lung cancer after induction chemotherapy and concurrent chemoradiotherapy (ESPATUE). J Clin Oncol 2015;33:4194-201.

16. Pless M, Stupp R, Ris HB, et al. Induction chemoradiation in stage IIIA/N2 non-small-cell lung cancer: a phase 3 randomised trial. Lancet 2015;386:1049-56.

17. Thomas M, Rube C, Hoffknecht P, et al. Effect of preoperative chemoradiation in addition to preoperative chemotherapy: a randomised trial in stage III non-smallcell lung cancer. Lancet Oncol 2008;9:636-48.

18. Katakami N, Tada H, Mitsudomi T, et al. A phase 3 study of induction treatment with concurrent chemoradiotherapy versus chemotherapy before surgery in patients with pathologically confirmed N2 stage IIIA nonsmall cell lung cancer (WJTOG9903). Cancer 2012;118:6126-35.

19. Girard N, Mornex F, Douillard JY, et al. Is neoadjuvant chemoradiotherapy a feasible strategy for stage IIIA-N2 non-small cell lung cancer? Mature results of the randomized IFCT-0101 phase II trial. Lung Cancer 2010;69:86-93.

20. Guo SX, Jian Y, Chen YL, et al. Neoadjuvant chemoradiotherapy vesus chemotherapy alone followed by surgery for resectable stage iii non-small-cell lung cancer: a meta-analysis. Sci Rep 2016;6:34388.

21. Yang CF, Kumar A, Gulack BC, et al. Long-term outcomes after lobectomy for non-small cell lung cancer when unsuspected pN2 disease is found: a National Cancer Data Base analysis. J Thorac Cardiovasc Surg 2016;151:1380-8.

22. Ramnath N, Dilling TJ, Harris LJ, et al. Treatment of stage III non-small cell lung cancer: Diagnosis and management of lung cancer, 3rd ed: American College of Chest Physicians evidence-based clinical practice guidelines. Chest 2013;143:e314S-40S.

23. Postmus PE, Kerr KM, Oudkerk M, et al. Early and locally advanced non-small-cell lung cancer (NSCLC): ESMO Clinical Practice Guidelines for diagnosis, treatment and follow-up. Ann Oncol 2017;28:iv1-21.

24. Hellmann MD, Chaft JE, William WN Jr, et al. Pathological response after neoadjuvant chemotherapy in resectable non-small-cell lung cancers: proposal for the use of major pathological response as a surrogate endpoint. Lancet Oncol 2014;15:e42-50.

25. Cottrell TR, Thompson ED, Forde PM, et al. Pathologic features of response to neoadjuvant anti-PD-1 in resected 
non-small-cell lung carcinoma: a proposal for quantitative immune-related pathologic response criteria (irPRC). Ann Oncol 2018;29:1853-60.

26. Planchard D, Popat S, Kerr K, et al. Metastatic non-small cell lung cancer: ESMO Clinical Practice Guidelines for diagnosis, treatment and follow-up. Ann Oncol 2018;29:iv192-237.

27. Gandhi L, Rodriguez-Abreu D, Gadgeel S, et al. Pembrolizumab plus chemotherapy in metastatic nonsmall-cell lung cancer. N Engl J Med 2018;378:2078-92.

28. Govindan R, Szczesna A, Ahn MJ, et al. Phase III trial of ipilimumab combined with paclitaxel and carboplatin in advanced squamous non-small-cell lung cancer. J Clin Oncol 2017;35:3449-57.

29. Langer CJ, Gadgeel SM, Borghaei H, et al. Carboplatin and pemetrexed with or without pembrolizumab for advanced, non-squamous non-small-cell lung cancer: a randomised, phase 2 cohort of the open-label KEYNOTE-021 study. Lancet Oncol 2016;17:1497-508.

30. Paz-Ares L, Luft A, Vicente D, et al. Pembrolizumab plus chemotherapy for squamous non-small-cell lung cancer. $\mathrm{N}$ Engl J Med 2018;379:2040-51.

31. Socinski MA, Jotte RM, Cappuzzo F, et al. Atezolizumab for first-line treatment of metastatic nonsquamous NSCLC. N Engl J Med 2018;378:2288-301.

32. Rizvi NA, Hellmann MD, Brahmer JR, et al. Nivolumab in combination with platinum-based doublet chemotherapy for first-line treatment of advanced non-small-cell lung cancer. J Clin Oncol 2016;34:2969-79.

33. Gadgeel S, Rodríguez-Abreu D, Speranza G, et al. Updated analysis from KEYNOTE-189: pembrolizumab or placebo plus pemetrexed and platinum for previously untreated metastatic nonsquamous non-small-cell lung cancer. J Clin Oncol 2020;38:1505-17.

34. Emens LA, Middleton G. The interplay of immunotherapy and chemotherapy: harnessing potential synergies. Cancer Immunol Res 2015;3:436-43.

35. Galluzzi L, Zitvogel L, Kroemer G. Immunological mechanisms underneath the efficacy of cancer therapy. Cancer Immunol Res 2016;4:895-902.

36. Charoentong P, Finotello F, Angelova M, et al. Pancancer immunogenomic analyses reveal genotypeimmunophenotype relationships and predictors of response to checkpoint blockade. Cell Rep 2017;18:248-62 .

37. Blank CU, Rozeman EA, Fanchi LF, et al. Neoadjuvant versus adjuvant ipilimumab plus nivolumab in macroscopic stage III melanoma. Nat Med 2018;24:1655-61.

38. Cloughesy TF, Mochizuki AY, Orpilla JR, et al.
Neoadjuvant anti-PD-1 immunotherapy promotes a survival benefit with intratumoral and systemic immune responses in recurrent glioblastoma. Nat Med 2019;25:477-86.

39. Liu J, Blake SJ, Yong MC, et al. Improved efficacy of neoadjuvant compared to adjuvant immunotherapy to eradicate metastatic disease. Cancer Discov 2016;6:1382-99.

40. Forde PM, Chaft JE, Smith KN, et al. Neoadjuvant PD-1 blockade in resectable lung cancer. N Engl J Med 2018;378:1976-86.

41. Bott MJ, Yang SC, Park BJ, et al. Initial results of pulmonary resection after neoadjuvant nivolumab in patients with resectable non-small cell lung cancer. J Thorac Cardiovasc Surg 2019;158:269-76.

42. Sepesi B, Cascone T, William W, et al. OA13.06 Surgical outcomes following neoadjuvant nivolumab or nivolumab plus ipilimumab in non-small cell lung cancer NEOSTAR study. J Thorac Oncol 2019;14:S241-2.

43. Cascone T, William WN, Weissferdt A, et al. Neoadjuvant nivolumab $(\mathrm{N})$ or nivolumab plus ipilimumab (NI) for resectable non-small cell lung cancer (NSCLC): Clinical and correlative results from the NEOSTAR study. J Clin Oncol 2019;37:abstr 8504.

44. Oezkan F, He K, Owen D, et al. OA13.07 Neoadjuvant atezolizumab in resectable NSCLC patients: immunophenotyping results from the interim analysis of the multicenter trial LCMC3. J Thorac Oncol 2019;14:S242-3.

45. Gao S, Li N, Gao S, et al. Neoadjuvant PD-1 inhibitor (Sintilimab) in NSCLC. J Thorac Oncol 2020;15:816-26.

46. Provencio M, Nadal E, Insa A, et al. OA13.05 NADIM study: updated clinical research and outcomes. J Thorac Oncol 2019;14:S241.

47. Shu CA, Grigg C, Chiuzan C, et al. Neoadjuvant atezolizumab + chemotherapy in resectable non-small cell lung cancer (NSCLC). J Clin Oncol 2018;36:abstr 8532.

48. Rothschild S, Zippelius A, Eboulet EI, et al. SAKK 16/14: Anti-PD-L1 antibody durvalumab in addition to neoadjuvant chemotherapy in patients with stage IIIA(N2) non-small cell lung cancer (NSCLC)_A multicenter single-arm phase II trial. J Clin Oncol 2020;38:abstr 9016.

49. Caushi J, Ji Z, Zhang J, et al. P2.04-24 Transcriptional profiling of neoantigen specific t cells in resectable NSCLC treated with neoadjuvant anti-PD-1.J Thorac Oncol 2019;14:S717.

50. Anagnostou V, Forde PM, White JR, et al. Dynamics of tumor and immune responses during immune checkpoint 
blockade in non-small cell lung cancer. Cancer Res 2019;79:1214-25.

51. Reuben A, Zhang J, Lin HY, et al. T cell repertoire analysis of non-small cell lung cancer patients treated with neoadjuvant nivolumab alone or in combination with ipilimumab (NEOSTAR trial). J Clin Oncol 2019;37:abstr 8532.

52. Laza-Briviesca R, Cruz-Bermudez A, Casarrubios M, et al. Immune cell biomarkers on neo-adjuvant chemoimmunotherapy treatment for resectable stage IIIA NSCLC patients. Ann Oncol 2019;30:v507.

Cite this article as: Palmero R, Vilariño N, Navarro-Martín A, Nadal E. Induction treatment in patients with stage III nonsmall cell lung cancer. Transl Lung Cancer Res 2021;10(1):539554. doi: $10.21037 /$ tlcr-20-420 